\begin{tabular}{|c|c|}
\hline Title & Causal Role of Noradrenaline in the Timing of Internally Generated Saccades in Monkeys \\
\hline Author(s) & Suzuki, Tomoki W.; Tanaka, Masaki \\
\hline Citation & $\begin{array}{l}\text { Neuroscience, 366, 15-22 } \\
\text { https://doi.org/10.1016/.neuroscience.2017.10.003 }\end{array}$ \\
\hline Issue Date & 2017-12-16 \\
\hline Doc URL & http:/hdl.handle.net/2115/2163 \\
\hline Rights & $\begin{array}{l}\text { (0) 2017. This manuscript version is made available under the CC-BY-NC-ND } 4.0 \text { license } \\
\text { https:/creativecommons.org/icenses/by-nc-nd/4.0/ }\end{array}$ \\
\hline Rights(URL) & https://reativecommons.org/icenses/by-nc-nd/4.0/ \\
\hline Type & article (author version) \\
\hline File Information & Neuroscience366_15.pdf \\
\hline
\end{tabular}

Instructions for use 


\section{Causal role of noradrenaline in the timing of internally-generated saccades in monkeys}

Tomoki W. Suzuki \& Masaki Tanaka

Department of Physiology, Hokkaido University School of Medicine, Sapporo 060-8638, Japan

Text pages: 18

Figures: 4

Table: 1

Abstract: 200 words

Correspondence to:

Dr. Masaki Tanaka

Department of Physiology

Hokkaido University School of Medicine

North 15, West 7, Sapporo 060-8638, Japan

Tel: +81 11-706-5039, Fax: +81 11-706-5041

E-mail:masaki@med.hokudai.ac.jp 


\section{Highlights}

- Macaque monkeys were trained to report the passage of $1 \mathrm{~s}$ following a visual cue by making a self-timed saccade.

- A selective noradrenaline reuptake inhibitor (reboxetine) delayed self-timed but not visually-triggered saccades.

- Reboxetine did not alter the proportion of impulsive, immediate saccades to the cue.

- Other parameters remained unchanged except for the accuracy of visually-triggered saccades in the high dose condition.

\section{Abstract}

We recently found that when monkeys performed an oculomotor version of the time production task, the trial-by-trial latency of self-timed saccades was negatively correlated with pupil diameter just before the delay period (Suzuki et al., 2016). Since pupil diameter has been shown to correlate with neuronal activity in the locus coeruleus, the level of noradrenaline (NA) in the brain might regulate the subjective passage of time. To examine this, we orally administered a selective noradrenaline reuptake inhibitor (reboxetine, $0.4-0.8 \mathrm{mg}$ ) when animals made a self-initiated memory-guided saccade $>1 \mathrm{~s}$ following the appearance of a brief visual cue. We found that reboxetine delayed self-timed saccades, while the latency of visually-triggered saccades remained unchanged. Because the changes in proportions and latencies of early impulsive saccades were comparable between conditions with and without drug administration, alteration of self-timing might not result from reduced impulsivity. We also assessed other behavioral parameters (saccade accuracy, velocity, and latency variance), but failed to find any drug effect except for the accuracy of visually-triggered saccades in the high dose condition, indicating that reboxetine specifically altered self-timing under our experimental conditions. Our results suggest that NA-related internal states may causally regulate temporal information processing in the brain.

\section{Keywords}

saccade; temporal information processing; interval timing; noradrenaline; neuromodulator; non-human primate

\section{Abbreviations}

NA, noradrenaline; FP, fixation point; fMRI, functional magnetic resonance imaging;

LC, locus coeruleus; DA, dopamine; ADHD, attention deficit hyperactivity disorder 


\section{Introduction}

Time flies quickly when we are having fun but goes by slowly when we are bored. Distortions of the sense of time have been demonstrated under a variety of experimental conditions (Wittmann, 2009). However, how internal states such as attention, arousal and affective state alter our time experience remains elusive. Since temporal information processing relies on many different networks in the brain (Ivry and Schlerf, 2008; Merchant et al., 2013), a slight change in neuronal transmission throughout the brain may have a substantial impact on the subjective passage of time. Neuromodulators may play a key role because they are closely related to internal states and modulate the functional links between global brain circuits (Corbetta et al., 2008; Brezina, 2010; Sara and Bouret, 2012; Marder, 2014). For example, transient depletion of dopamine has been reported to reduce functional connectivity in the cortico-striatal pathways during set shifting in humans (Nagano-Saito et al., 2008), while serotonergic stimulation causes a widespread decrease in resting state connectivity between many cortical and subcortical areas except for the thalamus and the cerebellum (Schaefer et al., 2014; Klaassens et al., 2017). Furthermore, recent functional imaging studies demonstrated that systemic application of selective noradrenaline (NA) reuptake inhibitors led to a global reorganization of functional connectivity in multiple cortical and subcortical networks (van Wingen et al., 2014; Metzger et al., 2016; Guedj et al., 2016; van den Brink et al., 2016) that was associated with improved behavioral performance (Grefkes et al., 2010; Rae et al., 2017).

One way to probe the internal state is to measure pupil diameter (Laeng et al., 2012; Sirois and Brisson, 2014). Previous studies have shown that pupil diameter is strongly correlated with the level of NA signaling in the brain (Aston-Jones and Cohen, 2005; Murphy et al., 2014; Varazaani et al., 2015; Joshi et al., 2016). We recently found in monkeys that the report of elapsed time in a time production task could be predicted by pupil diameter early in the trial (Suzuki et al., 2016), suggesting that the level of endogenous NA might influence the subjective passage of time.

NA is closely related to a variety of cognitive processes including arousal, attention and mood (Chamberlain et al., 2006, Sara and Bouret, 2012). To date, only a few studies suggested a role for NA in temporal information processing (Rammsayer et al. 2010, Penney et al., 1996). However, its diverse functions regulating internal factors 
led us to reason that NA may be involved in the neuronal processes that link internal states and temporal information processing. In the current study, to elucidate the roles of NA in temporal information processing, we trained monkeys to report the passage of $1 \mathrm{~s}$, and assessed the effects of systemic administration of reboxetine, a selective NA reuptake inhibitor.

\section{Experimental procedures}

Animal preparation and surgical procedures

Three adult Japanese monkeys (Macaca fuscata, two males and one female, 6-8 kg, 613 years old, monkeys B, D and F) were used. All experimental protocols were evaluated and approved in advance by the Hokkaido University Animal Care and Use Committee. In two separate surgeries, each animal was implanted with a head holder and an eye coil under general isoflurane anesthesia using sterile techniques (Tanaka, 2005). Opiate and nonsteroidal analgesics were administered during the surgery and a few days later. After the animals had fully recovered from the surgery, they were trained on the oculomotor tasks. During the training and experimental sessions, monkeys sat in a primate chair with their heads fixed to the chair in a darkened booth. Horizontal and vertical eye position were recorded using the search coil technique (MEL-25, Enzanshi Kogyo).

Visual stimuli and behavioral tasks

Experiments were controlled by a real-time data acquisition system (TEMPO, Reflective Computing). Visual stimuli were presented on a 27-inch liquid crystal display (XL2720Z, BenQ, refresh rate: $60-144 \mathrm{~Hz}$ ) located $46 \mathrm{~cm}$ from the eyes $\left(66 \times 40^{\circ}\right.$ of visual angle). In the self-timed saccade task (Fig. 1A), each trial began with the appearance of a fixation point (FP, blue $0.7^{\circ}$ square) located at the center of the screen. Fixation was detected when eye position remained within $3^{\circ}$ of the FP for $400 \mathrm{~ms}$. After an additional fixation period (800, 1050, or $1300 \mathrm{~ms}$, randomly selected), a visual cue (white $0.7^{\circ}$ square) was presented $15^{\circ}$ right or left of the FP for $100 \mathrm{~ms}$. To obtain reward, the animals were required to generate a memory-guided saccade to the cue location after the mandatory delay interval (1000 ms) following the cue onset. Because the FP disappeared only after the saccade, the animals needed to internally decide 
saccade timing without any explicit Go signal. Saccades were detected online when eye position deviated $>4-5^{\circ}$ from the FP. Trials were aborted if monkeys did not maintain fixation until the cue onset or failed to generate a saccade until $1700 \mathrm{~ms}$ following the visual cue, although the latter trials were vanishingly rare $(0.15 \%)$. Each correct trial ended with a delivery of juice reward. Cue location remained unchanged throughout each experimental session.

In 39 out of 43 experimental sessions, visually-guided saccade trials (Fig. 1B) were randomly interleaved (20\% of the trials). In this task, the FP differed in color (red $0.7^{\circ}$ square) from the self-timed saccade task, and the animals were required to make an immediate saccade ( $<700 \mathrm{~ms}$ ) to the target that appeared at the time of FP offset. We used an unusually long time window for correct performance in order to include possible delayed response following drug administration; however, visually-guided saccades with latencies $>400 \mathrm{~ms}$ were rare $(0.57 \%)$.

\section{Drug administration}

Approximately 1-1.5 $\mathrm{h}$ after the animals started to perform the task, a selective NA reuptake inhibitor (reboxetine, Pfizer, 0.4 or $0.8 \mathrm{mg}$ dissolved in $2 \mathrm{~mL}$ water with $0.1 \mathrm{~g}$ sucrose) was administered orally. The dose was determined based on the therapeutic dose for the treatment of depression ( $4 \mathrm{mg}$ twice a day) and the previous functional magnetic resonance imaging (fMRI) studies in humans (Grefkes et al., 2010; Metzger et al., 2015). We compared the data between the pre-dose trials during a 30-min period before drug administration and the post-dose trials during a 60-min period starting from 30 min after drug administration. In the non-drug control sessions, $0.1 \mathrm{~g}$ sucrose in 2 $\mathrm{mL}$ water only was administered. The order of drug conditions (control, 0.4 and $0.8 \mathrm{mg}$ reboxetine) was randomized except for monkey B. For this animal, most of the high dose experiments were performed in earlier sessions while control experiments were intermingled randomly throughout sessions. To avoid possible accumulating effects, we never administered reboxetine on successive days. The experimenter was not blind to the type of drug conditions.

\section{Data acquisition and analysis}

Eye position data were digitized at 16-bit resolution and sampled at $1 \mathrm{kHz}$ along with the event timestamps, and were saved in files during experiments for offline analyses 
that were performed using MATLAB (Mathworks). Trials with goal non-directed saccades ( $>7^{\circ}$ from cue/target location, 5.7\%) were excluded from the analysis. In the self-timed task, data with early impulsive saccades ( $<600 \mathrm{~ms}$ from the cue onset) were analyzed separately from the remaining trials (Fig. 2A, see Results; Suzuki et al., 2016). In the visually-guided saccade task, trials with early anticipatory saccades (reaction time $<70 \mathrm{~ms}, 0.06 \%$ ) were excluded from further analysis. For within-session analysis, we compared median saccade latency using a Wilcoxon rank sum test. For across-session analysis, we used a paired $t$-test to compare the means of median latency, or a one-way ANOVA to compare the difference in latency among conditions. We also computed the effect size (Cohen's $d$ ) defined as $\left(\mu_{\text {post }}-\mu_{\text {pre }}\right) / \operatorname{sqrt}\left[\left(\sigma_{\text {post }}^{2}+\sigma_{\text {pre }}{ }^{2}\right) / 2\right]$, where $\mu$ and $\sigma$ indicated the mean and standard deviation of saccade latency in each condition, respectively.

\section{Results}

Figure $2 A$ shows the distribution of saccade latency in a representative experiment. Two distinct populations of latencies were observed for both the pre-dose (black lines) and the post-dose data of $0.4 \mathrm{mg}$ reboxetine (red). We defined the trials with short latencies (<600 ms) as "impulsive" trials and analyzed them separately. When we compared the data from the remaining "self-timed" trials (Fig. 2B), saccade latency was clearly delayed after administration of reboxetine (median [interquartile range] = 1060 [998, 1150] versus 1137 [1081, 1249] ms, $n=149$ and 287 trials, Wilcoxon rank sum test, $p<$ $10^{-11}$ ). Since impulsivity is linked with temporal information processing (Wittmann and Paulus, 2008), changes in self-timing might be attributed to a decrease of impulsivity following reboxetine application. However, we failed to find significant difference in the occurrence of impulsive saccades before and after drug administration $\left(\chi^{2}\right.$ test, $p=$ 0.70 ) in this session. Although impulsive saccade latencies were prolonged following drug application in this experiment (median [interquartile range] $=203$ [193, 217] versus 228 [211, 236] ms, $n=18$ and 39 trials, Wilcoxon rank sum test, $p<0.01$ ), similar changes in impulsive saccade latencies were found even in the control sessions with no reboxetine administration (see below).

These drug effects were consistent across sessions. Figure $3 A$ plots the distributions of self-timed saccade latencies during the post-dose period (relative to the 
median of pre-dose data) for 43 sessions in three animals (black, blue and red lines represent control, $0.4 \mathrm{mg}$ and $0.8 \mathrm{mg}$ reboxetine sessions, respectively). The distribution of saccade latencies following reboxetine administration differed significantly from that following only sucrose administration (control) in all six cases (two dose conditions for three monkeys, Kolmogorov-Smirnov test, $n=374-2442$ trials, $p<10^{-7}$ ). To quantify the effects on self-timing, we compared the median latencies between the trials before and after drug application in individual sessions (Fig. 3B). After reboxetine administration, self-timed saccade latencies significantly altered in 18 out of 24 experiments, and in most cases (83\%, 15/18) saccades were delayed (Wilcoxon rank sum test, $p<0.05,7 / 12$ and $8 / 12$ for 0.4 and $0.8 \mathrm{mg}$ sessions, respectively; Table 1 ). In contrast, following only sucrose administration (control), saccade latency remained unchanged in most experiments (11/19), whereas saccades were delayed (3/19) or facilitated (5/19) in a minority of sessions. In the population as a whole, self-timed saccade latency significantly altered following reboxetine administration (two-tailed paired $t$-test, $p<0.02$ for both doses), but not in the control sessions ( $p=0.43$ ).

To further elucidate drug effects on timing, we compared changes in median latency of self-timed saccades (Fig. 3C) with those of visually-guided saccades (Fig. $3 D$ ). For the self-timed trials, we found a significant difference among conditions (one-way ANOVA, $F_{2,40}=5.78, p<0.01$ ). Post hoc multiple comparisons indicated that both reboxetine groups significantly differed from control (Tukey-Kramer test, $p<$ $0.05)$, whereas the two dose groups did not differ from each other ( $p=0.83)$. However, for the visually-guided trials, we did not find any significant difference among dose conditions (one-way ANOVA, $F_{2,36}=1.43, p=0.25$ ), although one might see a weak trend in Figure $3 D$.

Similar results were obtained when we compared the effect size (Cohen's $d$, see Experimental procedures) between conditions. For self-timed saccades, the effect size averaged $-0.09 \pm 0.39(\mathrm{SD}, n=19$; median $=-0.06), 0.29 \pm 0.31(n=12$; median $=$ $0.28)$ and $0.30 \pm 0.34(n=12$; median $=0.36)$ for the control, 0.4 and $0.8 \mathrm{mg}$ reboxetine conditions, respectively, and these values differed significantly (one-way ANOVA, $F_{2,40}$ $=6.31, p<0.01)$. Post hoc multiple comparisons showed significant differences between the control and each of the two reboxetine conditions (Tukey-Kramer test, $p<$ $0.02)$, but not between different doses $(p=1.0)$. For visually-guided saccades, the 
values averaged $0.08 \pm 0.46(\mathrm{SD}, n=17$; median $=0.16), 0.23 \pm 0.37(n=11$; median $=$ $0.08)$ and $0.28 \pm 0.64(n=11$; median $=0.10)$ for the control, 0.4 and 0.8 mg conditions, respectively, and these values were not significantly different (one-way ANOVA, $F_{2,36}=$ $0.62, p=0.55)$. Thus, reboxetine delayed self-timed but not visually-guided saccades.

We also compared the changes in median latency separately for each animal. Considering the small number of sessions for each dose condition (Table 1), we combined the data for both reboxetine conditions and compared them with the control (sucrose only) condition. For self-timed trials, the changes in median latency in the reboxetine condition were significantly greater than those in the control condition for all three monkeys $(-1.3 \pm 20.3 \mathrm{~ms}$ versus $36.8 \pm 43.6 \mathrm{~ms},-68.7 \pm 41.5 \mathrm{~ms}$ versus $0.0 \pm$ $28.6 \mathrm{~ms}, 7.6 \pm 46.5 \mathrm{~ms}$ versus $50.8 \pm 32.0 \mathrm{~ms}$, for monkeys $\mathrm{B}$, D and F, respectively, two-tailed unpaired t-test, $p<0.05)$. In contrast, for visually-guided trials, we failed to find a significant reboxetine effect in any of the animals $(4.2 \pm 7.1$ ms versus $10.2 \pm$ $10.9 \mathrm{~ms},-5.7 \pm 3.5 \mathrm{~ms}$ versus $-4.1 \pm 5.3 \mathrm{~ms},-0.8 \pm 7.1 \mathrm{~ms}$ versus $3.6 \pm 9.2 \mathrm{~ms}$, for monkeys $\mathrm{B}, \mathrm{D}$ and $\mathrm{F}$, respectively, $p>0.24)$. Thus, the effects of reboxetine on self-timing were consistent among animals.

As most error trials were due to earlier self-timed saccades (e.g., Fig. 2A), increased latencies might reduce error trials. In other words, the potential link between latency and success rate may suggest the possibility that the changes in self-timing could merely reflect the alteration of behavioral strategy to earn more reward. However, we found that the changes in success rate among conditions were not consistent (mean \pm SD: $-4.2 \pm 6.0 \%, 1.0 \pm 7.5 \%, 0.2 \pm 6.2 \%$, for control, 0.4 and $0.8 \mathrm{mg}$ conditions, respectively, one-way ANOVA, $F_{2,40}=2.99, p=0.06$ ), while those in saccade latency were very consistent as described above. Furthermore, even when we selected sessions with no behavioral improvement (i.e., decreased correct rate during post-dose period; 15 , 5 and 6 sessions), we still observed a significant difference in the amount of changes in latency across conditions (mean $\pm \mathrm{SD}:-22.0 \pm 37.5 \mathrm{~ms}, 2.9 \pm 27.1 \mathrm{~ms}, 30.5 \pm 52.2 \mathrm{~ms}$, $F_{2,23}=3.87, p<0.05$ ), whereas the changes in correct rate were comparable (mean \pm SD: $\left.-6.0 \pm 5.2 \%,-5.4 \pm 2.5 \%,-4.5 \pm 3.0 \%, F_{2,23}=0.27, p=0.77\right)$. Thus, the change in latency was not necessarily concomitant with improved performance, indicating that the change in self-timed latency was unlikely to be a byproduct of the alteration of behavioral strategy to reduce error trials. 
Reboxetine failed to alter the proportion and latency of impulsive saccades. For most reboxetine experiments (10/12 for both doses), the proportion of impulsive saccades remained unchanged (Fisher's exact test, $p>0.05$ ). For 21 experiments with more than five impulsive trials (10, 5 and 6 sessions for control, 0.4 and $0.8 \mathrm{mg}$ conditions, respectively), the changes in the proportion of impulsive trials did not differ between the control and the reboxetine conditions (unpaired $t$-test, $t_{19}=0.23, p=0.82$ ). For 12 experiments with more than five impulsive trials during both the pre- and post-dose periods (5, 3 and 4 sessions for control, 0.4 and $0.8 \mathrm{mg}$ conditions, respectively), impulsive saccade latencies were generally prolonged during the post-dose period, irrespective of the drug conditions (4/5, 3/3, 2/4, Wilcoxon rank sum test, $p<0.05$ ). The overall changes in median latencies of impulsive saccades did not differ between the control and the reboxetine conditions (mean \pm SD: $15.6 \pm 4.8 \mathrm{~ms}$ versus $15.6 \pm 6.2 \mathrm{~ms}$, unpaired $t$-test, $t_{10}=0.01, p=0.99$ ). Thus, while the latencies of impulsive saccades were often prolonged during the post-dose period in all conditions, we found no significant effect of reboxetine on impulsivity in the present study.

To explore other possible effects of reboxetine, we also examined the coefficient of variation (CV) of saccade latency, saccade peak velocity and accuracy (distance between target and saccade endpoint). For these values, we computed the difference between the pre/post-dose periods in each session, and compared them across different conditions using one-way ANOVAs. For self-timed saccades, we failed to find any significant difference among dose conditions for all three variables (Figs. 4A-C, left panels, $F_{2,40}=0.80,2.09$ and 2.57, $p>0.05$ ). For visually-guided saccades, changes in $\mathrm{CV}$ of saccade latency and peak velocity did not differ among dose conditions (Figs. 4A and $B$, right panels, $F_{2,36}=1.18$ and 2.98, $p>0.05$ ), while those in accuracy showed a significant difference (Fig. $4 C$, right panel, $F_{2,36}=5.96, p<0.01$ ). Post hoc multiple comparisons indicated that the value for the $0.8 \mathrm{mg}$ condition was statistically greater than those for the other two conditions (Tukey-Kramer test, $p<0.05$ ). When we compared these three parameters for impulsive saccades, we failed to find any significant difference (unpaired $t$-test, 5 and 7 sessions with more than five impulsive trials during both periods, $p>0.49$ ). These results demonstrated a sharp contrast with the consistent changes in self-timed saccade latency, suggesting that the effect of reboxetine was relatively specific to self-timing under our experimental condition. 


\section{Discussion}

In the current study we found that systemic application of a NA reuptake inhibitor (reboxetine) delayed self-timed saccades in monkeys. Since NA reuptake inhibitors can ameliorate impulsivity of attention deficit hyperactivity disorder (ADHD; Michelson et al., 2002), decreased impulsivity might reduce earlier saccades, resulting in the delay of self-timed saccades. However, changes in the proportion of early immediate saccades to the cue were comparable between sessions with and without reboxetine, suggesting that the alteration of self-timing following reboxetine administration did not result from decreased impulsivity. Furthermore, reboxetine did not alter the latency of either visually-guided saccades (Fig. 3D) or impulsive saccades, indicating that general lengthening of sensorimotor processing for saccades could not account for the present results. In addition, we also failed to find any significant change in the variance of latency and the other saccade parameters, except for the accuracy of visually-guided saccades after administration of $0.8 \mathrm{mg}$ reboxetine (Fig. 4C). Thus, the delay of self-timing was almost the only effect of reboxetine in our experimental condition.

Recent functional imaging studies have shown that NA reuptake inhibitors induce reorganization of large-scale functional network in the brain in both humans and monkeys (Grefkes 2010; Metzger 2016; Guedj 2016; Rae et al., 2017). Given that temporal information processing relies on distributed networks involving multiple cortical and subcortical regions (Lewis and Miall, 2003; Mauk and Buonomano, 2004; Ivry and Schlerf, 2008, Merchant et al., 2013), alteration of self-timing following reboxetine administration is not entirely surprising. Indeed, Rammsayer et al. (2010) previously showed that reboxetine improved duration discrimination with a 1000 -ms standard interval in humans. Furthermore, Penney et al. (1996) found that both agonist and antagonist of $\alpha 2$-adrenoceptor altered self-timing in the order of $10 \mathrm{~s}$ in rats. Nevertheless, to the best of our knowledge, the present study is the first to quantitatively demonstrate that a transient increase of NA signaling prolongs self-timing in the time production task in the range of $1 \mathrm{~s}$. We expect that the present results in eye movements could be generalized to the conditions requiring other types of movements such as manual response.

We recently found in monkeys that pupil size was negatively correlated with 
trial-by-trial latency of self-timed saccades, showing that larger pupil diameter predicted shorter saccade latency (Suzuki et al., 2016). Because pupil diameter and neuronal activity in the locus coeruleus (LC) exhibit a positive correlation (Aston-Jones and Cohen, 2005; Murphy et al., 2014; Varazaani et al., 2015; Joshi et al., 2016), reboxetine might be expected to increase endogenous NA and facilitate self-timing (Faber, 2017). However, in this study we obtained the opposite results. There are several possible reasons for this discrepancy.

First, the effects of NA signaling on self-timing might differ for different time scales. While in the previous study we assessed the trial-by-trial correlation between pupil size and self-timing, in the current study we compared data between the pre- and post-dose periods. Although reboxetine may shift the global functional network from one state to another, pupil size might only reflect moment-by-moment fluctuation of NA signals in a given condition. In this regard, our previous findings that pupil diameter correlated with self-timing relative to the behavioral goal imposed by the task-rule rather than the absolute timing (Suzuki et al., 2016) might be relevant.

When we compare the data between the two periods, it should be noted that some adaptive changes in behavioral strategy might occur. Specifically, in our self-timed task, delayed saccades could result from a strategy to earn more reward. Although our results contradict the view that the alteration of saccade latency resulted from improved performance, we cannot fully exclude this possibility. For example, the drug effects could be interpreted in terms of inhibitory control (Chamberlain et al., 2006). We found that the latency of visually-guided saccades and the latency and occurrence of impulsive saccades did not alter following administration of reboxetine, and these results clearly ruled out the general increase of inhibitory control. However, it is still possible that cognitive control specifically inhibiting internally-generated movement might be involved.

Second, it has been shown that NA reuptake inhibitors elevate the dopamine (DA) level in the prefrontal cortex, where DA transporters are only sparsely expressed and therefore DA clearance from extracellular space also depends on NA transporters (Carboni et.al., 1990; Bymaster et.al., 2002; Swanson et.al, 2006). Because implication of DA in temporal processing has been established (Buhusi and Meck, 2005; Coull et al., 2012; Parker et al., 2014; Soares et al., 2016; Kunimatsu and Tanaka, 2016), the present 
results of delayed self-timing might be partly mediated by the increased DA in the prefrontal cortex. Because different neuromodulators often work cooperatively (Brezina, 2010; Marder, 2014), the possible enhancement of both NA and DA signals may be important.

Finally, reboxetine might differently alter the phasic and tonic components of NA signaling, which, respectively, are related to the event-evoked transient and baseline activity of neurons in the LC (Aston-Jones and Cohen, 2005). Because these NA signals are thought to mediate distinct functions, the discrepancy between the current and the previous studies might be related to the different effects of reboxetine on the two types of NA signals. For example, reboxetine might alter only the tonic signals, while pupil size might reflect the phasic signals. We notice that these hypotheses are highly speculative and will require further verification in future studies.

Although our results clearly demonstrated a close relationship between neuromodulatory signaling and self-timing, systemic drug application limited the inference on the underlying neural mechanisms. Nevertheless, because the role of the cortico-basal ganglia circuitry in time production is well documented (Buhusi and Meck, 2005; Bartolo et al., 2014; Emmons et al., 2016; Kunimatsu and Tanaka, 2016), it is possible that reboxetine alters neuronal processing in the relevant circuits. Although direct NAergic projections to the basal ganglia are only sparse (Swanson and Hartman, 1975; Jones and Yong, 1985; Baldo et al., 2003), a previous fMRI study demonstrated that NA reuptake inhibitors strongly modulated neural signals within the cortico-basal ganglia pathways (Easton et al., 2007). Furthermore, Ohta et al. (2016) recently reported that NA and adrenoceptor agonists altered the response gain of striatal neurons to excitatory input. Another line of evidence also suggests a possible role of cortico-cerebellar networks in the NA-dependent regulation of self-timing observed in the present study. A number of studies have implicated the cerebellum in self-timing (Ivry and Schlerf, 2008; Ashmore and Sommer, 2013), especially in its fine adjustment (Ohmae et al., 2017). Moreover, the cerebellum has been found to receive massive NAergic innervation (Bloom et. al., 1971; Olson and Fuxe 1971; Nyström et al., 1972; Yamamoto et al., 1977).

The present results indicate the potential involvement of NA in the processes linking our internal states and self-timed movements. Nonetheless, we should note that 
possible increase of DA in the prefrontal cortex might have a substantial role in the present findings. It is also true that the results could be interpreted in terms of adaptive changes in behavioral strategy or higher order cognitive control rather than temporal information processing as discussed above. Future studies with more sophisticated behavioral paradigms and regionally specific manipulation of NA signals in experimental animals will address these unresolved issues.

\section{Acknowledgements}

The authors thank T. Mori, A. Hironaka, and H. Miyaguchi for their assistance with animal care and surgery; M. Suzuki for administrative help; M. Takei and Y. Hirata in the Equipment Developing Group at the Research Institute for Electronic Science for manufacturing some of the equipment; and all lab members for helpful comments and discussions. This work was supported by grants from the Ministry of Education, Culture, Sports, Science and Technology of Japan, the Takeda Science Foundation, and the Uehara Memorial Foundation. The authors declare no competing commercial interest. 


\section{References}

Ashmore RC, Sommer MA (2013) Delay activity of saccade-related neurons in the caudal dentate nucleus of the macaque cerebellum. J Neurophysiol 109:2129-2144.

Aston-Jones G, Cohen JD (2005) An integrative theory of locus coeruleus-norepinephrine function: adaptive gain and optimal performance. Annu Rev Neurosci 28:403-450.

Bartolo R, Prado L, Merchant H (2014) Information processing in the primate basal ganglia during sensory-guided and internally driven rhythmic tapping. J Neurosci 34:3910-3923.

Brezina V (2010) Beyond the wiring diagram: signalling through complex neuromodulator networks. Philos Trans R Soc Lond B Biol Sci 365:2363-2374.

Buhusi CV, Meck WH (2005) What makes us tick? Functional and neural mechanisms of interval timing. Nat Rev Neurosci 6:755-765.

Bymaster FP, Katner JS, Nelson DL, Hemrick-Luecke SK, Threlkeld PG, Heiligenstein JH, Morin SM, Gehlert DR, Perry KW (2002) Atomoxetine increases extracellular levels of norepinephrine and dopamine in prefrontal cortex of rat: a potential mechanism for efficacy in attention deficit/hyperactivity disorder. Neuropsychopharmacology 27:699-711.

Carboni E, Tanda GL, Frau R, Di Chiara G (1990) Blockade of the noradrenaline carrier increases extracellular dopamine concentrations in the prefrontal cortex: evidence that dopamine is taken up in vivo by noradrenergic terminals. J Neurochem 55:1067-1070.

Chamberlain SR, Müller U, Robbins TW, Sahakian BJ (2006) Neuropharmacological modulation of cognition. Curr Opin Neurol 19:607-612.

Corbetta M, Patel G, Shulman GL (2008) The reorienting system of the human brain: from environment to theory of mind. Neuron 58:306-324.

Coull JT, Hwang HJ, Leyton M, Dagher A (2012) Dopamine precursor depletion impairs timing in healthy volunteers by attenuating activity in putamen and supplementary motor area. J Neurosci 32:16704-16715.

Emmons EB, Ruggiero RN, Kelley RM, Parker KL, Narayanan NS (2016) Corticostriatal field potentials are modulated at delta and theta frequencies during interval-timing task in rodents. Front Psychol 7:459.

Faber NJ (2017) Neuromodulation of pupil diameter and temporal perception. J Neurosci $37: 2806-2808$.

Grefkes C, Wang LE, Eickhoff SB, Fink GR (2010) Noradrenergic modulation of cortical networks engaged in visuomotor processing. Cereb Cortex 20:783-797.

Guedj C, Monfardini E, Reynaud AJ, Farnè A, Meunier M, Hadj-Bouziane F (2016) Boosting norepinephrine transmission triggers flexible reconfiguration of brain networks at rest. Cereb Cortex.

Ivry RB, Schlerf JE (2008) Dedicated and intrinsic models of time perception. Trends Cogn Sci 12:273-280.

Joshi S, Li Y, Kalwani RM, Gold JI (2016) Relationships between pupil diameter and neuronal activity in the locus coeruleus, colliculi, and cingulate cortex. Neuron 89:221-234. 
Klaassens BL, Rombouts SA, Winkler AM, van Gorsel HC, van der Grond J, van Gerven JM (2017) Time related effects on functional brain connectivity after serotonergic and cholinergic neuromodulation. Hum Brain Mapp 38:308-325.

Kunimatsu J, Tanaka M (2016) Striatal dopamine modulates timing of self-initiated saccades. Neuroscience 337:131-142.

Laeng B, Sirois S, Gredebäck G (2012) Pupillometry: A window to the preconscious? Perspect Psychol Sci 7:18-27.

Lewis PA, Miall RC (2003) Distinct systems for automatic and cognitively controlled time measurement: evidence from neuroimaging. Curr Opin Neurobiol 13:250-255.

Marder E, O'Leary T, Shruti S (2014) Neuromodulation of circuits with variable parameters: single neurons and small circuits reveal principles of state-dependent and robust neuromodulation. Annu Rev Neurosci 37:329-346.

Mauk MD, Buonomano DV (2004) The neural basis of temporal processing. Annu Rev Neurosci 27:307-340.

Merchant H, Harrington DL, Meck WH (2013) Neural basis of the perception and estimation of time. Annu Rev Neurosci 36:313-336.

Metzger CD, Wiegers M, Walter M, Abler B, Graf H (2015) Local and global resting state activity in the noradrenergic and dopaminergic pathway modulated by reboxetine and amisulpride in healthy subjects. Int J Neuropsychopharmacol 19:pyv080.

Michelson D, Allen AJ, Busner J, Casat C, Dunn D, Kratochvil C, Newcorn J, Sallee FR, Sangal RB, Saylor K, West S, Kelsey D, Wernicke J, Trapp NJ, Harder D (2002) Once-daily atomoxetine treatment for children and adolescents with attention deficit hyperactivity disorder: a randomized, placebo-controlled study. Am J Psychiatry 159:1896-1901.

Murphy PR, O'Connell RG, O'Sullivan M, Robertson IH, Balsters JH (2014) Pupil diameter covaries with BOLD activity in human locus coeruleus. Hum Brain Mapp 35:4140-4154.

Nagano-Saito A, Leyton M, Monchi O, Goldberg YK, He Y, Dagher A (2008) Dopamine depletion impairs frontostriatal functional connectivity during a set-shifting task. J Neurosci 28:3697-3706.

Nyström B, Olson L, Ungerstedt U (1972) Noradrenaline nerve terminals in human cerebral cortices: first histochemical evidence. Science 176:924-926.

Ohmae S, Kunimatsu J, Tanaka M (2017) Cerebellar roles in self-timing for sub- and supra-second intervals. J Neurosci 37:3511-3522.

Ohta H, Kohno Y, Arake M, Tamura R, Yukawa S, Sato Y, Morimoto Y, Nishida Y, Yawo H (2016) Adrenergic receptor-mediated modulation of striatal firing patterns. Neurosci Res 112:47-56.

Olson L, Fuxe K (1971) On the projections from the locus coeruleus noradrealine neurons: the cerebellar innervation. Brain Res 28:165-171.

Parker KL, Chen KH, Kingyon JR, Cavanagh JF, Narayanan NS (2014) D1-dependent 4 Hz oscillations and ramping activity in rodent medial frontal cortex during interval timing. J Neurosci 34:16774-16783. 
Penney TB, Holder MD, Meck WH (1996) Clonidine-induced antagonism of norepinephrine modulates the attentional processes involved in peak-interval timing. Exp Clin Psychopharmacol 4:82-92.

Rae CL, Nombela C, Rodríguez PV, Ye Z, Hughes LE, Jones PS, Ham T, Rittman T, Coyle-Gilchrist I, Regenthal R, Sahakian BJ, Barker RA, Robbins TW, Rowe JB (2016) Atomoxetine restores the response inhibition network in Parkinson's disease. Brain 139:2235-2248.

Rammsayer TH, Hennig J, Haag A, Lange N (2001) Effects of noradrenergic activity on temporal information processing in humans. Q J Exp Psychol B 54:247-258.

Sara SJ, Bouret S (2012) Orienting and reorienting: the locus coeruleus mediates cognition through arousal. Neuron 76:130-141.

Schaefer A, Burmann I, Regenthal R, Arélin K, Barth C, Pampel A, Villringer A, Margulies DS, Sacher J (2014) Serotonergic modulation of intrinsic functional connectivity. Curr Biol 24:2314-2318.

Sirois S, Brisson J (2014) Pupillometry. Wiley Interdiscip Rev Cogn Sci 5:679-692.

Suzuki TW, Kunimatsu J, Tanaka M (2016) Correlation between pupil size and subjective passage of time in non-human primates. J Neurosci 36:11331-11337.

Swanson CJ, Perry KW, Koch-Krueger S, Katner J, Svensson KA, Bymaster FP (2006) Effect of the attention deficit/hyperactivity disorder drug atomoxetine on extracellular concentrations of norepinephrine and dopamine in several brain regions of the rat. Neuropharmacology 50:755-760.

Tanaka M (2005) Involvement of the central thalamus in the control of smooth pursuit eye movements. J Neurosci 25:5866-5876.

van den Brink RL, Pfeffer T, Warren CM, Murphy PR, Tona KD, van der Wee NJ, Giltay E, van Noorden MS, Rombouts SA, Donner TH, Nieuwenhuis S (2016) Catecholaminergic neuromodulation shapes intrinsic MRI functional connectivity in the human brain. J Neurosci 36:7865-7876.

van Wingen GA, Tendolkar I, Urner M, van Marle HJ, Denys D, Verkes RJ, Fernández G (2014) Short-term antidepressant administration reduces default mode and task-positive network connectivity in healthy individuals during rest. Neuroimage 88:47-53.

Varazzani C, San-Galli A, Gilardeau S, Bouret S (2015) Noradrenaline and dopamine neurons in the reward/effort trade-off: a direct electrophysiological comparison in behaving monkeys. J Neurosci 35:7866-7877.

Wittmann M (2009) The inner experience of time. Philos Trans R Soc Lond B Biol Sci 364:1955-1967.

Wittmann M, Paulus MP (2008) Decision making, impulsivity and time perception. Trends Cogn Sci 12:7-12.

Yamamoto T, Ishikawa M, Tanaka C (1977) Catecholaminergic terminals in the developing and adult rat cerebellum. Brain Res 132:355-361. 


\section{Figure captions}

Figure 1. Behavioral paradigms. $\boldsymbol{A}$, Sequence of events in the self-timed saccade task. During central fixation, a visual cue appeared for $100 \mathrm{~ms}$. Monkeys were required to generate a self-timed memory-guided saccade toward the cue location $>1000 \mathrm{~ms}$ afterwards. The fixation point (FP) disappeared only after the saccade. $\boldsymbol{B}$, In the visually-guided saccade task, animals made an immediate saccade to the target in response to the FP offset. Note that color of the FP differed between the tasks.

Figure 2. Effects of noradrenaline (NA) reuptake inhibitor (reboxetine, $0.4 \mathrm{mg}$ ) on self-timing in a single experimental session. $\boldsymbol{A}$, Distribution of saccade latency in the self-timing task. Black and red lines denote the data for the pre-dose $(n=167)$ and post-dose $(n=326)$ trials, respectively. The vertical dashed line represents the latency border $(600 \mathrm{~ms})$ to discriminate impulsive from self-timed trials. Animals were rewarded only for trials with latency $>1000 \mathrm{~ms}$ (vertical solid line). B, Cumulative relative frequency of self-timed saccade latencies for the data shown in A. Median saccade latencies differed significantly (Wilcoxon rank sum test, $p<10^{-11}$ ).

Figure 3. Summary of multiple experiments. $\boldsymbol{A}$, Distribution of self-timed saccade latency during the post-dose period for monkeys B ( $n=6160)$, D $(n=1451)$ and F $(n=$ 4923). For each session, latencies were subtracted from the median latency during the pre-dose period. Different colors represent different doses of reboxetine ( 0.4 and 0.8 $\mathrm{mg}$ ). Only sucrose was orally administered in the control sessions (black dashed lines). $\boldsymbol{B}$, Comparison of median latencies between the pre-dose and post-dose periods in individual experiments. Filled symbols denote sessions exhibiting a significant difference (Wilcoxon rank sum test, $p<0.01$ ). For the population, reboxetine significantly altered saccade latency (two-tailed paired t-test, $p<0.02$ for both reboxetine conditions). $\boldsymbol{C , D}$, Comparison of the changes in median latency among different conditions for self-timed $(C, n=43)$ and visually-guided $(D, n=39)$ trials. For each condition, vertically aligned dots indicate data from the same animal. Note that vertical scales differ between the tasks. A one-way ANOVA revealed a significant drug effect on the latency of self-timed saccades only $(C)$. Asterisks indicate the pairs with a significant difference according to post-hoc multiple comparisons (Tukey-Kramer test, ${ }^{*} p<0.05$ and $\left.{ }^{* *} p<0.01\right)$. 
Figure 4. Comparison of the changes in CV of saccade latency $(\boldsymbol{A})$, peak velocity $(\boldsymbol{B})$ and saccade accuracy $(\boldsymbol{C})$ across different conditions for each task. Each box-whisker plot represents the median, quantiles, and the range of the data. X's indicate the mean values. Asterisk indicates a significant difference revealed by post hoc multiple comparisons (Tukey-Kramer test, $p<0.05$ ). 
Table 1. Summary of the results in the self-timed task for each monkey.

\begin{tabular}{rccc}
\hline & & \multicolumn{2}{c}{ Reboxetine } \\
\cline { 3 - 4 } & Control & $0.4 \mathrm{mg}$ & $0.8 \mathrm{mg}$ \\
\hline Monkey B & & & \\
Session number & 8 (inc 0, dec 0) & 6 (inc 3, dec 0) & 6 (inc 4, dec 2) \\
Trial number (pre) & $174 \pm 13$ & $178 \pm 7$ & $171 \pm 20$ \\
(post) & $346 \pm 26$ & $347 \pm 21$ & $346 \pm 12$ \\
\% success rate (pre) & $83 \pm 9$ & $79 \pm 11$ & $80 \pm 18$ \\
(post) & $79 \pm 11$ & $81 \pm 7$ & $79 \pm 14$ \\
\hline Monkey D & & & $2(1,0)$ \\
Session number & $3(0,2)$ & $2(0,1)$ & $137 \pm 25$ \\
Trial number (pre) & $137 \pm 21$ & $145 \pm 33$ & $202 \pm 55$ \\
(post) & $232 \pm 35$ & $227 \pm 38$ & $87 \pm 1$ \\
\% success rate (pre) & $93 \pm 5$ & $86 \pm 10$ & $4(3,0)$ \\
(post) & $82 \pm 3$ & $80 \pm 8$ & $166 \pm 9$ \\
Monkey F & & & $318 \pm 12$ \\
Session number & $8(3,3)$ & $4(4,0)$ & $94 \pm 3$ \\
Trial number (pre) & $168 \pm 6$ & $172 \pm 1$ & $97 \pm 2$ \\
(post) & $302 \pm 52$ & $315 \pm 11$ & $93 \pm 6$ \\
\% success rate (pre) & $93 \pm 4$ & $96 \pm 2$ & \\
\hline
\end{tabular}

Numbers in parentheses denote the number of sessions showing a significant change in saccade latency following administration of reboxetine or only sucrose (Wilcoxon rank sum test, $p<$ 0.05), summarizing separately for the cases with increased (inc) or decreased (dec) latency. Each entry indicates mean \pm SD among sessions. Note the similar number of trials for each drug condition. 


\section{A Self-timed task}

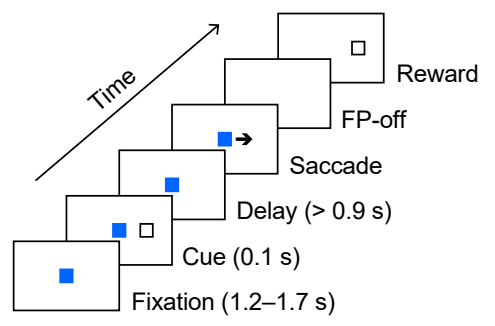

B Visually-guided task

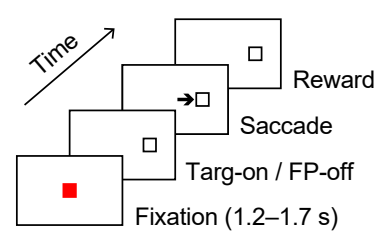

FIG.1 

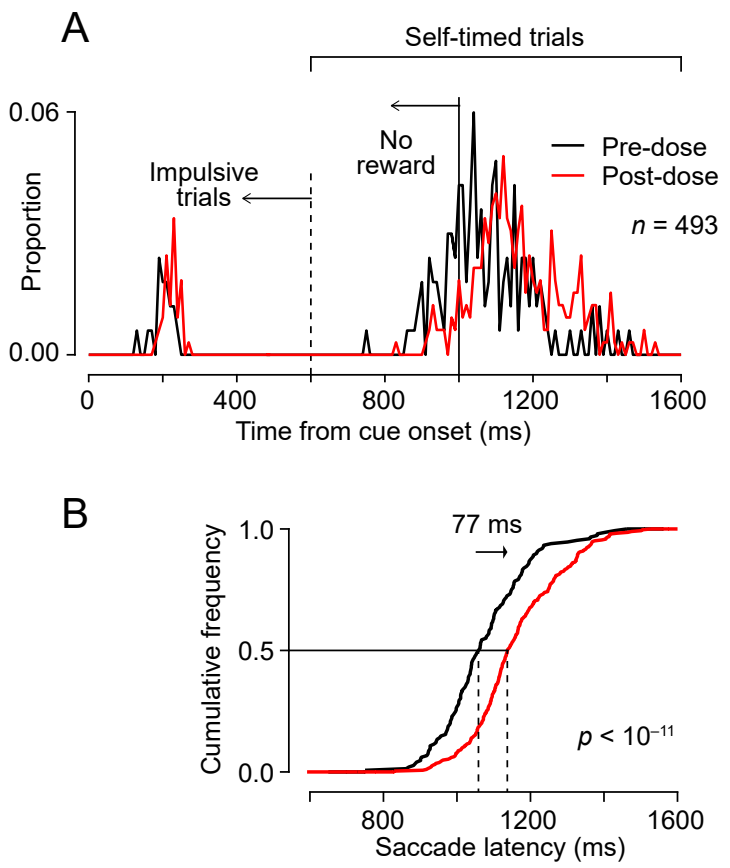

FIG.2 


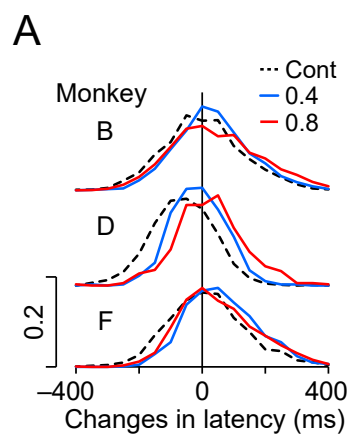

B
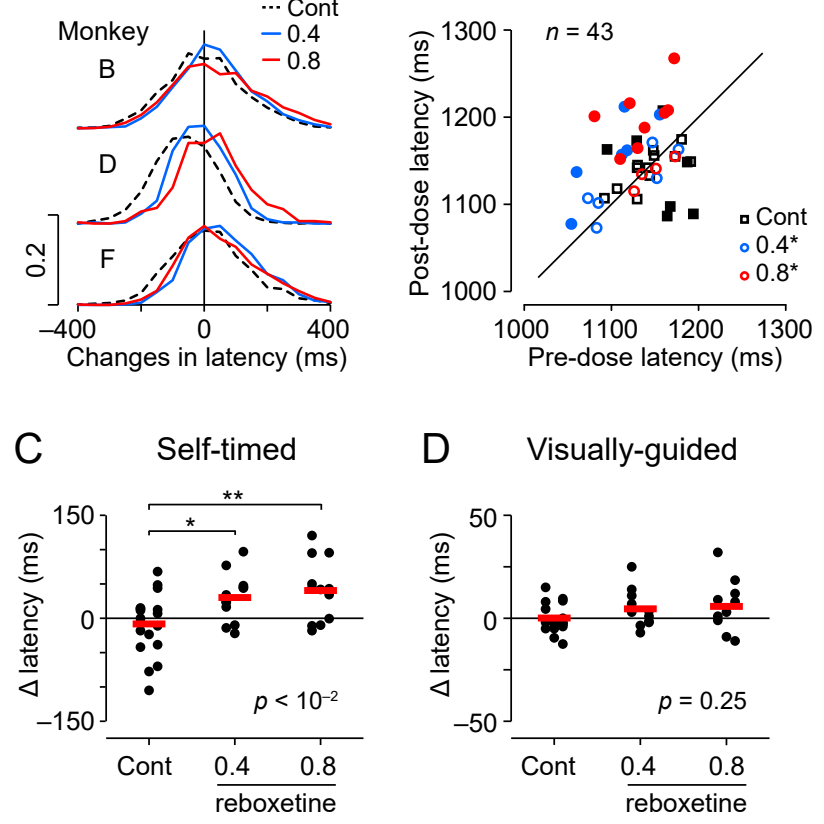

D Visually-guided

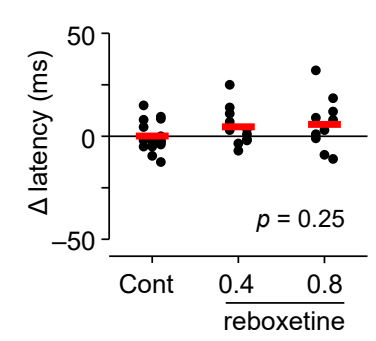

FIG.3 

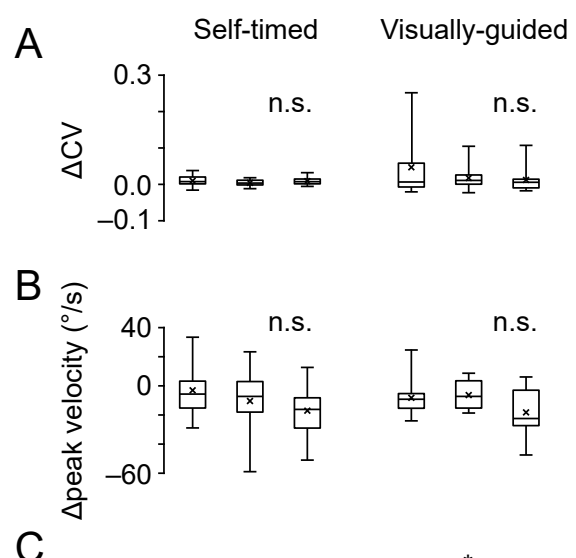

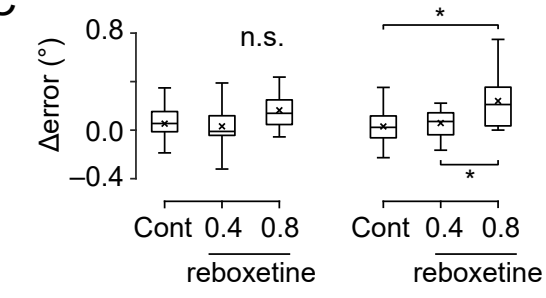

FIG.4 